\title{
Médiation et agencement : une poétique de la langue de spécialité
}

Jean-Jacques Lecercle

\section{(2) OpenEdition}

\section{Journals}

Édition électronique

URL : http://journals.openedition.org/asp/1734

DOI : 10.4000/asp. 1734

ISBN : 978-2-8218-0384-8

ISSN : 2108-6354

Éditeur

Groupe d'étude et de recherche en anglais de spécialité

Édition imprimée

Date de publication : 1 octobre 2001

Pagination : 1-17

ISSN : 1246-8185

\section{Référence électronique}

Jean-Jacques Lecercle, « Médiation et agencement : une poétique de la langue de spécialité », ASp [En ligne], 31-33 | 2001, mis en ligne le 22 septembre 2010, consulté le 01 mai 2019. URL : http:// journals.openedition.org/asp/1734; DOI : 10.4000/asp.1734

Ce document a été généré automatiquement le 1 mai 2019

Tous droits réservés 


\title{
Médiation et agencement : une poétique de la langue de spécialité
}

\author{
Jean-Jacques Lecercle
}

\section{Une parabole}

1 Je vais abuser de ma position de candide dans ce congrès. Il est notoire, en effet, que je ne suis pas spécialiste des langues de spécialité. Non que je ne m’y intéresse pas : je suis fier d'avoir dirigé l'habilitation de mon collègue et ami Vidalenc (1995), qui m'a fourni des aperçus précis et quasi exhaustifs sur deux langues de spécialité au moins, celle des scientifiques et celle des scénarios de films.Mais je vais profiter de la distance que j'ai visà-vis de votre discipline pour commencer par vous parler de tout autre chose. Cette autre chose sera ensuite qualifiée d'objet de parabole, pour me permettre de revenir aux questions de langue qui nous intéressent directement.

Si je commence par une parabole, c'est pour vous dire ce que j'entends par médiation, ou plutôt, puisque ce concept est résolument hégélien, par la dialectique du médiat et de l'immédiat. Le sujet de votre congrès suggère qu'en tant que corps constitué vous mettez votre idéal du moi dans le médiat plutôt que dans l'immédiat. Je vais essayer de vous dire pourquoi je pense que vous avez raison de le faire, mais j'emprunterai des chemins détournés. Voilà donc l'autre chose dont il sera d'abord question. C'est un tableau, une annonciation de Francesco del Cossa, peintre ferrarais de la seconde moitié du $15^{\mathrm{e}}$ siècle, peinte pour une église de Bologne, et que l'on peut voir aujourd'hui au musée de Dresde (voir annexe 1). Si je devais dire d'un mot ce qui caractérise le style de cette œuvre, et ce qui m'attire en elle, je dirais : l'exubérance de la représentation, la joie de la perspective entièrement maîtrisée et de la profondeur récemment découverte. Ce tableau n'a pas encore recours aux contorsions du maniérisme, mais il a déjà perdu la simplicité et le hiératisme des primitifs et de Piero della Francesca. Tableau éminemment décoratif, ce qui était une caractéristique notoire des peintres de Ferrare, proche en cela des tableaux de Crivelli, contemporain de Cossa, il nous présente le monde dans sa richesse 
foisonnante et surtout dans ses trois dimensions : la surface de la toile est une frontière immatérielle que notre regard est avec insistance invité à franchir.

Cette exubérance de la représentation a quelque chose à voir avec l'immédiateté : le tableau est en réalité plus présentation, sur le mode de l'immédiat, que représentation d'une scène trop connue. L'organisation en profondeur des plans, la complexité de l'architecture, la position des personnages, même si leur mouvement est arrêté sur image, produisent un fort effet visuel de présence. Cet effet de présence immédiate s'accompagne, même aujourd'hui, d'un effet de réel. Il suffit de comparer ce tableau soit avec une icône byzantine sur le même sujet, soit avec une peinture gothique, telle l' Annonciation de Simone Martini, qui se trouve aux Offices : chez Martini, la ligne qui unit l'ange à la Vierge court à la surface du tableau et la matérialise (au point que les paroles de l'ange, qui sont inscrites sur cette ligne, qui va de la bouche de l'ange à l'oreille de la Vierge - souvenez-vous que cette conception est auriculaire - sont sculptées en relief sur la surface du tableau) ; chez Cossa, cette ligne est une diagonale, qui parcourt et institue la profondeur de l'espace du tableau, produisant un effet de réel, c'est-à-dire de trompel'œil. Et ces corps dispersés en profondeur, par leur attitude, par leurs traits, par les vêtements qui les couvrent, produisent une impression de réalité, dont Martini n'a cure. Chez Cossa, même les ailes de l'ange, ces objets notoirement impossibles, ont l'apparence d'ailes d'oiseau.

On peut aller plus loin. Le tableau de Cossa n'est pas seulement réaliste, il est, si vous me pardonnez l'anachronisme, embourgeoisé; un certain effet de reconnaissance y est produit. Cet effet est, en quelque sorte, naturel; nous avons toujours déjà vu une représentation de l'Annonciation. Mais il est ici appuyé : cette vierge est une femme réelle, dont le modèle arpentait sans doute les rues de Ferrare ; cet ange est un beau jeune homme ; cette architecture d'arrière-plan est irréelle, mais aisément reconnaissable (que diable vient faire ici cette tour en ruine? Mais je n'ai aucun mal à l'identifier). Tout au fond, sous l'auréole de l'ange, on distingue même une porte médiévale, que deux moines s'apprêtent à franchir. Au premier plan les marmi finti sont, malgré leur feintise, plus vrais que nature. Et, sous la main gauche de l'ange, se promène un chien, détail qui suffit à ancrer la scène dans la réalité quotidienne contemporaine.

5 Cette Annonciation, plus présentation que représentation, lieu d'effets de réel et de reconnaissance, se caractérise donc par son immédiateté : un ange passe, avec arrêt sur image.

6 Et pourtant, il apparaît assez vite que cette immédiateté est de surface, qu'elle passe par une série de médiations. Il apparait en effet très vite que l'immédiatement présent ne se soutient que de l'impossible. Dans son analyse de ce tableau, Daniel Arasse (1999) fait remarquer que l'architecture devrait s'effondrer faute de seconde colonne qui, à la place exacte qu'occupe le spectateur, devrait soutenir l'architrave qui, en haut du tableau, se dirige vers nous. Il fait également remarquer qu'il y a dans le tableau une autre diagonale qui croise la première (laquelle construit la présence immédiate de la scène), celle qui va de Dieu, minuscule parce que lointain (c'est-à-dire soumis, de façon inhabituelle, aux lois de la perspective humaine) à l'escargot qui, en bas à droite, rampe sur le bord du tableau, et qui, si on le rapporte au pied de l'ange, est de taille démesurée : cet escargot est long, nous dit Arasse, de dix-huit centimètres.

7 Nous avons donc affaire à une première médiation, qui est celle du signe. L'escargot, dont on pensait à l'époque qu'il s'autofécondait, est le symbole de la Vierge. Il est aussi le signe du bord du tableau, qu'il marque de sa présence, rappelant à la fois qu'une frontière 
infranchissable sépare la réalité intrapicturale et la réalité extrapicturale du monde, et invitant le regard du spectateur à la franchir. Sa taille démesurée marque cette fonction antiréaliste, parce que soit symbolique, soit métapicturale. Et une fois l'escargot vu, le tableau se fait forêt de symboles. Cette colonne, qui occupe le centre du tableau et s'impose à l'attention du spectateur, ce n'est pas, bien sûr, seulement une colonne. C'est la marque de la séparation ontologique entre l'ange et la Vierge : l'ange ne peut envahir l'espace marial de la chambre et de son seuil - dans l'Annonciation de Martini, cette séparation est marquée par un vase contenant des lys. Mais c'est aussi, c'est du moins ce que le commentaire d'Arasse m'a appris, le symbole du Christ qui est en cet instant conçu (la colombe de l'Esprit Saint vient d'être lâchée). Il n'est pas jusqu'aux deux arches qui ne fassent signe : pourquoi y en a-t-il deux, et ni une ni trois ? Parce qu'elles inscrivent le $\mathrm{M}$ de Marie dans le tableau, bien sûr. Il est donc clair que nous ne sommes plus du tout dans le domaine de l'immédiat, mais dans le domaine de la médiation par excellence, celui du signe ou du symbole.

En réalité nous n'y avons jamais été, car la scène est toujours déjà peuplée de signes : même vue, c'est toujours déjà une scène écrite. Car la scène originaire, qui est représentée ici, n’a jamais été présentée ou vue, non seulement parce qu'elle est mythique (rien ne nous interdit de croire à ce mythe), mais parce qu'elle est toujours déjà médiée par le texte des Evangiles qui la narre et qui est son vrai lieu d'origine. C'est bien pour cela que la Vierge de l'Annonciation est toujours représentée lisant (ce que n'indiquent pas les Evangiles - un évangile apocryphe, ou est-ce une invention des libres-penseurs des Lumières, suggère qu'elle était en train de ravauder les chausses de Joseph). Pour indiquer sa grande piété, bien sûr, et pour nous rappeler que l'Annonciation était ellemême annoncée dans les prophéties de l'Ancien Testament, mais aussi, je le crois du moins, parce que cette scène, qui s'origine dans un livre, est une scène du Livre : ce qui s'incarne dans Marie, c'est l'esprit de la lettre.

On a donc une chaîne de médiations: un texte sacré, dont ce tableau d'autel est l'illustration. Un texte médié par une image, sous ses deux aspects d'istoria (qui met en histoire la narration, c'est-à-dire l'inscrit dans deux moments qui se répondent en miroir : le moment palestinien du mythe, et le moment italien de la scène représentée) et le disegno, c'est-à-dire la composition, qui produit les effets de réel et de reconnaissance pour ce qu'ils sont, autrement dit des effets : les paroles qui, contrairement à Martini, ne sont pas inscrites sur le tableau sont néanmoins présentes, non parce qu'elles volent dans l'atmosphère diaphane, mais parce qu'elles sont inscrites dans le geste de l'ange ('Ave'), dans les arcades qui surplombent la Vierge ('Maria'), et dans la colonne qui matérialise, fort matériellement, le fruit de cette conversation, et qui fonctionne comme une sorte de signature.

10 Et il y a une médiation supplémentaire, qui parachève la dialectique du médiat et de l'immédiat - et ici je me sépare définitivement de l'analyse d'Arasse : celle de l'abyme. Déjà, nous avons vu que l'escargot était signe métapictural. Mais regardez bien l'auréole de l'ange : c'est clairement un disque de métal fixé sur sa tête par une armature qui la transforme en casque. Cet ange, qui ressemble à un beau jeune homme, est un beau jeune homme qui joue un ange. Cette scène, qui est si réelle qu'elle ressemble à un décor, est donc en effet un décor, une scène à l'italienne : le tableau est virtuellement entouré par les rideaux d'un théâtre. Ces spectateurs que nous sommes sont donc bien des spectateurs : nous assistons à la mise en scène d'une scène, à la représentation d'une représentation. Nous sommes d'ailleurs présents aussi dans le tableau, sous la forme de la 
femme et de l'enfant au balcon, au-dessus de la tête de l'ange. Voilà donc pourquoi cette architecture est à la fois irréelle et réaliste : c'est un décor de théâtre, comme l'est cette architecture imaginaire parfois attribuée à Piero della Francesca, et que l'on peut voir au musée d'Urbino. La ville est un théâtre, la scène religieuse un drame : l'immédiateté a radicalement disparu, ou plutôt elle se donne enfin pour ce qu'elle est: une illusion soigneusement, par médiation, construite. La dialectique de l'immédiat et du médiat a donc, dans ce tableau, la forme suivante : il y a une chaîne de la médiation, qui va de la scène mythique au texte sacré, puis à l'illustration qu'est le tableau, puis au décor de théâtre que cette illustration, en abyme, reproduit, dans lequel la scène originale est mise en scène; et il y a, par boucle, un effet d'immédiateté, qui unit les deux termes de la chaîne, la scène mythique originelle et la mise en scène abyssale. Cet effet d'immédiateté, de mise en scène, construit bien sûr une illusion: dans notre tableau, elle est forte, et a nom trompe l'œil, effet de présence, de réel, de reconnaissance.

\section{Où diable est la langue dans tout cela ?}

11 La réponse à cette question est simple : les disciplines du langage (j'entends par là la critique littéraire autant que linguistique) sont fréquemment victimes d'une illusion d'immédiateté, par quoi il ne faut pas entendre nécessairement qu'elles s'imaginent avoir directement accès, par quelque intuition mystique, à l'essence du langage, mais qu'elles prétendent retrouver le fondement, l'origine directe, de la langue ou du sens. Je vous en propose deux exemples.

12 Le premier est emprunté à la linguistique. C'est, excusez du peu, le programme de recherches chomskyen. Je n'ai pas besoin de vous faire un dessin: c'est le plus prestigieux, le plus répandu (dans le monde entier), celui qui prétend être le plus scientifique. Cette prétention doit être contestée, en ce qu'elle est source d'une illusion d'immédiateté. Le chomskysme se présente en effet comme un naturalisme, qui réduit, au moins idéalement, les phénomènes linguistiques à des connexions physiques dans ce que Chomsky appelle le mind/brain. Les lois de la grammaire universelle sont des modèles abstraits de phénomènes biologiques ou psychologiques encore, provisoirement, inconnus, ce qui veut dire qu'un jour la linguistique s'effacera devant la biologie du cerveau, lorsque celle-ci aura suffisamment progressé. Ce réductionnisme, que le vieux marxiste que je suis a envie de qualifier de matérialisme vulgaire, sombre dans l'illusion d'immédiateté en ce qu'il postule une causalité directe du physique au linguistique, incarnée dans ce monstre de langues, mind/brain (on lui opposera, à titre de comparaison, le parallélisme spinoziste entre les deux modes de la substance ou la théorie de la « supervenience » des phénomènes mentaux chez Davidson).

Dans la doctrine chomskyenne, cette illusion d'immédiateté a des manifestations, et des conséquences délétères. Cela commence par la disparition de tout facteur externe (non que les chomskyens refusent, bien sûr, de reconnaître leur existence, mais ils leur dénient toute pertinence) : ainsi la grammaire universelle n'est pas objet d'apprentissage, étant innée. L'argument, unique et sans cesse ressassé, s'appuie sur la soi-disant explosion de compétence chez le jeune enfant : une connaissance complexe acquise si rapidement que la seule explication possible est de considérer qu'elle était toujours-déjà là, inscrite dans le cerveau comme une propriété de l'espèce. Je fais simplement remarquer que la structure de cet argument est la même que celle de l'argument cosmologique pour prouver l'existence de Dieu : l'horloge du monde est trop compliquée pour ne pas avoir 
été conçue par un horloger. Cela continue (mais sur ce point Chomsky est fidèle à Saussure) avec le postulat de l'arbitraire du signe (qui exclut ou marginalise les phénomènes d'iconicité) et le principe de synchronie (qui sort la langue de l'histoire, et ne réintroduit celle-ci que sous la forme, toujours dévalorisée, de la diachronie). Chez Chomsky, ces deux postulats saussuriens sont poussés jusqu'à l'extrême.

Le pire pour moi, dans cette illusion d'immédiateté, n'est pas là : il est dans la disparition des langues nationales en tant qu'objet d'étude pertinent. Car si l'on a accès immédiatement à la Langue, ou à la Grammaire universelle, le niveau médiat, ou intermédiaire, de la langue naturelle, ou nationale, perd tout intérêt : quelques switches de plus ou de moins dans le mind/brain, et l'on passe sans solution de continuité de l'allemand au hollandais, puis à l'anglais. Comme j'ai depuis toujours un intérêt passionné pour la langue anglaise, pour sa structure mais aussi pour son histoire et pour la culture qu'elle véhicule, vous comprendrez que cette affirmation d'immédiateté, qui est illusion et terrorisme scientiste, ne me réjouisse pas.

Et où sont les langues de spécialité dans ce modèle? La réponse est immédiate: nulle part. Si on a immédiatement accès à la Grammaire universelle (je caricature : je feins d'oublier la médiation du modèle abstrait, de la théorie), la langue de spécialité est doublement impertinente, en tant que liée à une langue naturelle, et que liée à un registre de cette langue, historiquement et culturellement constitué. Car la langue de spécialité suppose la médiation de l'historique et du social, comme le suppose tout point de vue " externe » au sens où Bourdieu entend cette expression, sur la langue. S'il existe des langues de spécialité, et un champ d'étude spécifique, c'est parce qu'il n'est pas de phénomène linguistique qui ne soit médié par l'historique, le culturel et le social - c'est-àdire par des institutions, des rituels et des pratiques, dont il est sinon impossible, du moins extrêmement réducteur et dangereux, de l'abstraire.

Mon second exemple est emprunté à la critique littéraire. Il n'est cependant pas si éloigné des préoccupations de la linguistique, car il concerne la construction du sens dans le texte littéraire. Ici, l'illusion d'immédiateté prend la forme d'une relation directe non pas tant entre le texte et son sens (tout sens ne se donne pas immédiatement), mais entre le texte et l'origine de son sens. Certains textes ont besoin d'un ouvre-boîte (j'ai fait la description de cette théorie de l'interprétation que j'appelle le tin-opener dans mon dernier livre, Interpretation as Pragmatics, 1999), mais une fois découvert ou recouvré, ce sens est directement c'est-à-dire causalement, lié à sa source. Les opinions quant à cette source varient : pour la pragmatique anglo-saxonne, c'est l'intention de sens du locuteur ou de l'auteur du texte, des conceptions plus anciennes de la littérature feront appel aux caractéristiques éternelles de la nature humaine (les chomskyens ne sont pas les seuls à y croire), aux valeurs éthiques qui inspirent le texte, à la psychologie qu'il met en scène. Je ne dis pas, naturellement, que ces derniers aspects ne sont pas présents dans le texte littéraire (pour ce qui est de la nature humaine, étant marxiste, j'ai du mal), mais que ce sont des effets d'immédiateté, des constructions médiates d'une illusion forte, mais illusoire. On doit lire Jane Austen comme si les personnages étaient des amis de la famille, comme si les situations dans lesquelles ils évoluent impliquaient les mêmes questions éthiques qui se posent dans notre vie quotidienne ; mais on doit aussi, et en même temps, savoir que ces effets sont médiatisés (par le genre du texte, par la construction de l'intrigue, par les fils textuels qui tissent les personnages, par le langage dans lequel seul ils existent). 
17 Tel était, bien entendu, l'objet de ma parabole, dans laquelle le tableau peut être considéré comme un texte. Je me suis appuyé sur la lecture que fait Arasse (dans laquelle le disegno révèle l'istoria et les valeurs religieuses qu'elle transmet : la seule médiation ici est que le disegno crypte le sens, les signes doivent être interprétés, mais leur interprétation provoque l'illumination du sens enfin directement atteint). Mais en remarquant un détail, l'auréole métallique de l'ange, qu'il néglige, j'ai essayé de montrer que l'istoria et ses valeurs étaient en réalité le résultat d'une mise en scène, d'une chaîne de médiations.

18 Je vous propose un exemple littéraire caricatural, et contre lequel j'ai déjà tonné en chaire. Push, roman de Sapphire (1996), a eu un grand succès il y a quelques années aux États-Unis et en France, où sa traduction s'est vendue à vingt mille exemplaires. Le roman est l'histoire d'une adolescente, noire, pauvre, obèse, de QI faible, ayant beaucoup de retard dans ses études. Et comme cela ne suffit pas, elle est mère célibataire d'un enfant trisomique, et enceinte d'un second engendré par son père, qui l'a violée et lui a transmis le SIDA. Mais il y a, à mon sens, encore pire : elle trouve le salut à l'école en rencontrant un professeur compréhensif qui l'incite à devenir poète ; et elle s'appelle Precious Jones. "Jones» comme tout un chacun, mais précieux, néanmoins, comme chaque individu singulier. Il est facile de comprendre ce qui ne va pas dans cette caricature de politiquement correct, ce qui est tartiné si épais que cela provoque la nausée: c'est l'immédiateté de la revendication identitaire de l'héroïne en tant que victime. Le texte, qui n'est pas si mal écrit (Sapphire est poétesse de son état) disparaît sous le poids de l'affect immédiat, qui n'est en réalité qu'une médiation culturelle : Precious Jones n'existe pas, non tant en ce qu'elle est un personnage de fiction qu'en ce qu'elle est une abstraction, une composition de catégories sociologiques défavorisées. Je me souviens d'un dessin de Wolinski, dans L'Humanité, où une jeune personne se préparait à présenter à des parents fort bourgeois un fiancé qui ressemblait étrangement à Sammy Davis Jr : il était en effet noir, juif et borgne. Dans la bulle, elle lui disait : «s'il te plaît, chéri, ne lui dis pas tout de suite que tu es communiste ».

19 Vous me direz que je charge des moulins à vent. Il est clair que Push n'est pas un chef d'œuvre, que le moment du structuralisme nous a débarrassé de l'immédiateté éthique ou psychologique en littérature et, sous l'influence de la linguistique, forcés à nous intéresser à l'écriture du texte, à sa constitution grammaticale. Mais l'illusion d'immédiateté est vieille comme le monde, elle s'appuie sur des effets (de réel, etc.) qui sont sources d'affects forts, elle revient sans cesse, et au galop, sous diverses formes : la revendication d'identité (le texte exprime directement, et revendique, pour son auteur, ses personnages ou ses lecteurs, une quelconque identité), mais aussi la revendication d'autorité (le sens du texte appartient à son auteur, et la tâche du critique est de le recouvrer), ou encore la revendication de valeurs (nous assistons actuellement, en critique littéraire, à un retour en force de l'éthique, et le parcours de Todorov, de la structure à la morale, est significatif).

Cette illusion d'immédiateté littéraire est forte et vivace, parce qu'elle s'appuie sur une philosophie du sens, à laquelle la linguistique n'échappe pas toujours (je pense en particulier à la pragmatique anglo-saxonne) qui fait dépendre le sens de l'énoncé de l'intention de sens du locuteur et de sa reconnaissance par l'interlocuteur. Cette philosophie est exprimée dans un article célèbre de H. Paul Grice, « Meaning » (1989), où il définit ce qu'il appelle le « sens non-naturel », c'est-à-dire le sens produit par des signes qui sont des artefacts humains et non des symptômes naturels. Il ne m'est pas indifférent 
que le lexique philosophique de Grice soit, sur ce point, emprunté soit à l'éthique du philosophe américain Stevenson, soit à la seconde critique Kantienne.

\section{Au-delà de l'illusion}

Mon ambition est de suggérer une théorie du sens qui ne verse pas dans ces illusions, et qui donc fasse une place au concept de langue de spécialité (car nous avons vu que l'immédiateté du rapport entre texte et grammaire universelle ne laissait aucune place à la langue de spécialité, et il en est de même de l'immédiateté du sens à ses origines individuelles, psychologiques ou éthiques).

Pour cela, je vais partir d'un exemple canonique (cela fait à peine dix ans que je le ressasse) (chapitres 2 et 3, op. cit. 1999) une phrase simple : « Let him have it, Chris ».

Si j'aborde cette phrase possédé par la première illusion d'immédiateté, celle d'un rapport direct à la langue ou la grammaire universelle, j'aurai une analyse syntaxique simple, à la Chomsky, qui me dira que cette simple phrase est une phrase simple, à l'impératif, et que la structure profonde rétablit un pronom personnel sujet, " you », effacé en surface. Le problème est que la connaissance de la structure syntaxique ne me permet pas ici de remplir le programme de l'analyse linguistique, c'est-à-dire de passer du son au sens ; la phrase contient un certain nombre de variables, qui échappent à l'analyse syntaxique : embrayeurs (le «you » implicite), plus généralement ce qu'on appelle en anglais des « indexicals » ( him » et « it »), mots vagues ou de sens générique, comme « have ». Même le nom «Chris » est source d'incertitude en ce qu'il est épicène. Si bien que le seul mot dont le sens m'est immédiatement donné par le système de la langue est le verbe "let", marqueur grammatical de l'impératif.

Cette immédiateté du système échoue donc à me donner le sens de la phrase. Mais ma seconde illusion d'immédiateté, littéraire et linguistique à la fois, vient à la rescousse. Elle me permet la donation immédiate du sens par reconstruction de l'intention du locuteur. Le problème de l'analyse syntaxique chomskienne, me dit-elle, est qu'elle est trop abstraite : elle s'intéresse à des phrases, non à des énoncés attestés, et sa pratique de l'astérisque est notoirement désinvolte, voire terroriste (l'astérisque sert parfois à exclure des phrases que la théorie rejette mais que la pratique de la langue accueille volontiers). La pragmatique et la linguistique énonciative, elles, réintroduisent le contexte, ou la situation d'énonciation. En déplaçant le centre d'intérêt vers le locuteur ou le sujet d'énonciation, elles permettent aux embrayeurs et autres pronoms personnels de trouver leur référence, et de désambiguiser le sexe du ou de la prénommé(e) Chris. Imaginons un tel contexte : le père, Chris, la mère (qui est l'auteur de l'énoncé), le fils (« him »), et la banane («it»). Alors la valeur de « have » s'induit du reste, et avec elle le sens de la phrase. La mère excédée enjoint au père de donner la banane à la marmaille qui réclame: "For heaven's sake, let him have it, Chris». Encore faut-il que cette famille soit monorejetonne, et que la banane soit perceptivement saillante. Et même dans ce cas, qu'est-ce qui nous assure que le « have it » ne doit pas être entendu métaphoriquement et que la mère, non seulement excédée mais poussée à la violence, ne demande pas au père de flanquer au marmot une raclée?

Il apparait donc qu'il y a des expressions dont le sens est constitutivement vague, ou ambigu, et que « have it » est l'une d'entre elles. Cela apparaitra encore plus clairement si je vous livre le contexte réel de ma phrase. Elle est au cœur de l'affaire Bentley, fait divers 
anglais du début des années cinquante. Derek Bentley, âgé de dix-huit ans, épileptique, et à la limite de la débilité mentale, cambriole un entrepôt en compagnie de Chris, son complice, âgé de seize ans. Ils se font prendre. En état d'arrestation, Bentley, qui n'était pas armé, est accusé d'avoir crié à son complice encore libre, «Let him have it, Chris». Ce sur quoi Chris sortit de sa poche un revolver et tua le policier qui s'apprêtait à l'arrêter. Trop jeune, il n'encourait pas la peine de mort. Bentley qui avait l'âge, fut poursuivi pour "constructive malice», complicité d'assassinat, condamné à mort et, malgré les protestations, pendu. Ma phrase fut décisive pour l'accusation qui l'utilisa pour établir l'incitation au meurtre.

Le problème est qu'elle est radicalement ambiguë, et que la situation seule ne suffit pas à la désambiguiser, sous une interprétation qui est une décision, c'est-à-dire un jugement. Prise littéralement, elle peut vouloir dire : «donne le lui », où «it » renvoie au pistolet. Un Bentley résigné demande à son complice de se rendre. Entendue métaphoriquement, elle peut vouloir dire: "descends-le». Un Bentley vindicatif pousse son complice au meurtre. L'accusation opta pour la seconde solution et par le jugement final réussi à imposer ce sens comme le sens de la phrase. On voit ici que l'espoir d'une donation immédiate du sens est bien une illusion : il ne sert à rien en effet de demander à Bentley ce qu'il voulait dire, car il choisira toujours, pour sauver sa tête, l'interprétation innocente. Le sens est donc établi médiatement (il est construit lors d'une succession d'audiences, dans l'agôn judiciaire) et contre ce qu'on sait par avance être le « sens du locuteur", ici par principe déclaré non fiable, c'est-à-dire lui-même médiat. Incidemment, le sens de la phrase était si peu immédiat que Bentley a été, il y a deux ans (mais un peu tard) réhabilité : il semble bien qu'il n'ait jamais prononcé cette phrase, qui serait une invention des policiers, désireux de trouver un coupable pour venger leur collègue. Comme vous le constatez, les médiations s'accumulent et se compliquent.

Je reconnais volontiers que la situation que j'ai décrite est singulière, et même exceptionnelle. Mais je pense que mon énoncé est exemplaire, et qu'il a valeur canonique. Il nous montre - ce qui peut et doit être généralisé - que le passage du son au sens ne se fait ni par référence directe au système de la langue, ni par appel immédiat à l'intention du sens du locuteur. Il nous montre, et ce ne sont pas les littéraires qui me contrediront, que le sens est toujours obtenu par construction, c'est-à-dire est toujours l'objet d'une interprétation, toujours atteinte par le biais de médiations.

Décrire ces médiations, c'est élaborer une théorie du sens en tant qu'il est contraint a) par le jeu de langage au sein duquel l'énoncé apparaît (vous reconnaissez ici une allusion à Wittgenstein tel qu'il fut relu, et approuvé, par Foucault) ; b) par la conjoncture au sein de laquelle il émerge, et dont le moment précis (c'est-à-dire non seulement le point d'émergence, mais la dynamique (momentum en anglais, mot qui a la même origine que " movimentum ») a un rapport causal avec l'énoncé et son sens (vous reconnaissez ici un thème hégélo-marxiste : je pense que nous avons besoin d'un concept de "conjoncture linguistique ») ; c) par la chaîne d'interpellation dans laquelle l'énoncé est pris, et qui va de l'institution (au sein de laquelle les jeux de langage sont pratiqués) au rituel (dont le jeu de langage est le versant langagier) à la pratique qui inscrit le rituel dans des actes (ceci est une théorie matérialiste du sens) et enfin à l'acte de langage en quoi consiste l'énoncé. Et vous avez reconnu la référence à la pragmatique anglo-saxonne (ou « quand dire, c'est faire ») combinée à la théorie althussérienne de l'idéologie comme interpellation d'individus en sujets. 

construit, et ce que ces médiations impliquent. Cet acte de langage, au singulier pouvoir performatif (puisqu'il est censé avoir causé la mort d'un policier, et qu'il a certainement causé celle de Bentley), loin d'être le résultat immédiat de l'intention de sens d'un sujet locuteur (son « auteur », je vous le rappelle, ne l'a jamais prononcé) est une construction, dans une conjoncture (la première conjoncture sécuritaire de l'après-guerre, où l'on craignait que les jeunes démobilisés ne sombrent dans la violence et la criminalité : le Garde des Sceaux refusa de gracier Bentley à cause de ces considérations politiques), dans une institution, l'institution judiciaire, avec ses rituels, ses pratiques, et ses jeux de langage (l'interprétation vraie de l'énoncé est ce que le jury décide). Et comme la phrase en question est, semble-t-il, une forgerie, les médiations se multiplient : c'est la police qui a placé ces mots dans la bouche de Bentley ; ils avaient été effectivement prononcés, dix ans plus tôt, dans des circonstances similaires, par un truand nommé Appleby, qui incitait son complice à liquider le policier (la phrase d'Appleby est : "Let him have it, he is alone " ce qui n'est guère ambigu); et ce truand avait vu trop de films de gangsters américains dont il imitait le parler rude mais pittoresque.

On comprend donc que la construction du sens d'un énoncé ne peut se satisfaire des illusions d'immédiateté, qu'elle doit les dénoncer comme des illusions. Bentley, locuteur postiche, est interpellé à sa place, celle du mort, par l'énoncé qui lui est attribué et dont le sens est construit par la conjoncture historique et son moment politique, par le jeu de langage dans le cadre duquel il est prononcé, par la chaîne d'interpellation qui lui donne naissance.

J'ai dans mon dernier livre (1999) proposé un modèle d'interprétation, c'est-à-dire de construction du sens, en ces termes. Il a nom modèle ALTER, d'après les cinq actants ou places structurelles qui le composent, Author, Language, Text, Encyclopaedia et Reader. C'est un modèle textualiste, conçu pour rendre compte de l'interprétation des textes littéraires (d'où les actants " auteur » et « lecteur ») mais qui prétend s'appliquer à toute situation d'interlocution (on remplacera alors «auteur» et «lecteur» par «locuteur» et « interlocuteur » ou « destinateur » et « destinataire »). Comme le montre le schéma, au centre du modèle il y a un énoncé, le texte, produit ou contraint par la langue et l'encyclopédie et interpellant à la place des actants auteur et lecteur des egos ou acteurs qui assurent soit la production, soit la réception du texte. Autour de ce texte, donc, la conjoncture linguistique construit du sens et interpelle des sujets.

Mais qu'est-ce que la « conjoncture linguistique ». C'est une conjonction de la conjoncture au sens marxiste habituel du terme (on parle de "conjoncture historique " et de «moment » de cette conjoncture - le moment par exemple où les slogans jusqu'ici justes deviennent faux, et où il faut en changer) et d'un état de la langue. Les deux éléments de la conjoncture sont notés $\mathrm{E}$ et $\mathrm{L}$. $\mathrm{E}$, c'est l'encyclopédie dans un sens dérivé de Eco : un ensemble de savoirs et de croyances, de discours publics tenus à un moment donné mais aussi les institutions, rituels et pratiques qui sont mis en discours et produisent des textes toujours collectifs, même si attribués à un auteur. Et L, c'est l'état de la langue dans une conjoncture: pas une grammaire universelle donc, pas l'inscription d'une faculté de l'espèce dans le psychologique et le biologique, pas donc un système abstrait, mais un état de langue, résultat d'une histoire, inscrit dans les rapports de force d'une conjoncture, systématique en ce sens qu'il s'impose à ses locuteurs (lesquels résistent, parfois avec succès, voyez l'histoire des pronoms épicènes en anglais), mais un système dynamique, soumis à une variation continue - la langue des sociolinguistes, de Voloshinov à Labov 
plutôt que celle de Saussure et de Chomsky. La conjoncture linguistique, c'est donc le cœur linguistique de mon modèle, LTE, l'origine des énoncés.

Et ces énoncés interpellent à leur place à nouveau frais dans chaque conjoncture, auteur et lecteur, A et R. Ce qui veut dire, ce que vous concéderez, qu'un texte change de lecteur chaque fois qu'il change de conjoncture, mais qu'il change aussi d'auteur - l'histoire des études shakespeariennes est l'histoire des Shakespeare par elle produit (naguère protestant, aujourd'hui catholique; mais aussi, et pourquoi pas, lacanien). Le sens est donc à la fois le produit d'une conjoncture linguistique et le résultat d'un processus d'interpellation. Ainsi Bentley, interpellé en auteur d'une phrase qu'il n'a jamais prononcée, n'est pas le même en 1951 (c'est un voyou, naturellement coupable, dont il faut faire un exemple pour dissuader ses congénères) et en 2001 (c'est la victime non d'une erreur, mais d'un acte de cruauté judiciaire : ce n'est plus un coupable, mais un mal-jugé : d'ailleurs, il a été réhabilité). Et le sens de l'énoncé n'est pas le même, même si les mots n'ont pas changé, en 1951 ou en 2001. En 1951 la phrase n'est pas ambiguë, ou plutôt son ambiguïté n'est que virtuelle (dans le système de la langue) et temporaire (le jury lui fixe son seul et vrai sens). En 2001, elle est radicalement ambiguë, construite pour être désambiguïsée par coup de force, par l'imposition d'un sens terroriste, légal mais illégitime. Et Bentley n'est plus qu'un des auteurs qu'elle interpelle : elle interpelle aussi Appleby, qui la prononça réellement, et les policiers anonymes, qui la mirent dans la bouche de Bentley. C'est d'ailleurs une des causes de sa réhabilitation. Des linguistes ont analysé les interrogations de Bentley par la police. Les phrases qui lui sont prêtées (il était illettré) sont d'une telle complexité et raideur administrative qu'il est impossible qu'il les ait proférées ou même comprises. Le système juridique anglais en a d'ailleurs depuis longtemps tiré des conséquences : vous comprenez pourquoi, chaque fois que l'inspecteur Morse, ou l'inspecteur Frost, interrogent un suspect, il y a, bien en évidence sur la table, un magnétophone.

\section{Et où diable est la langue de spécialité dans tout cela?}

Contrairement aux systèmes de linguistique théorique (qui nient toute pertinence aux jeux de langage ou aux registres ou bien s'en désintéressent), contrairement aux interprétations sémantico-littéraires (qui méprisent des textes trop collectifs, trop préconstruits ou trop contraints pour être intéressants), le modèle ALTER permet de faire aux langues de spécialité la place qui doit être la leur. Comme pour lui la langue est un agrégat de jeux de langage avec leurs règles ou leurs maximes, et comme la langue se combine à une encyclopédie (des institutions, des normes juridiques, des pratiques de groupe, etc.) il traite les langues de spécialité comme un de ses objets naturels, en ce qu'elles sont des jeux de langage, ou des familles de jeux de langage. Cela peut se dire d'une formule: pour le modèle ALTER, «il n'y a que des langues de spécialité ». La littérature est une famille de jeux de langage, c'est-à-dire une (ou des) langue (s) de spécialité. Cela nous permet de nous débarrasser du terrorisme de la linguistique théorique, qui méprise la linguistique appliquée (le langage des pilotes d'avion n'a peutêtre pas besoin de structures d'enchâssements syntaxiques : les voici donc détrônées de leur centralité théorique - on se souviendra que c'est sur de telles structures que Chomsky s'est appuyé pour nier toute pertinence au modèle des chaînes de Markov pour l'analyse des langues naturelles). Elle nous permet aussi de nous débarrasser du 
paternalisme de la critique littéraire. Non que, contrairement à mon prédécesseur à l'université de Cardiff, je prétende que le mode d'emploi d'une essoreuse, traduit en anglais du japonais, est un texte aussi complexe ou linguistiquement aussi riche que le monologue d'Hamlet. Mais je prétends que l'un et l'autre, en ce qu'ils participent de deux jeux de langage, relèvent du même niveau d'analyse : il faudra alors décrire précisément ces jeux de langage, qui n'obéissent pas aux mêmes maximes, ne relèvent pas des mêmes institutions et des mêmes pratiques, et n'ont donc pas les mêmes caractéristiques syntaxiques, sémantiques ou pragmatiques. Mais l'un et l'autre inscrivent une conjoncture linguistique et interpellent des individus en sujets. Autrement dit, et le mode d'emploi de l'essoreuse et le monologue de Shakespeare sont partie prenante de ce que Deleuze et Guattari (1980) appellent des "agencements collectifs d'énonciation », où ils trouvent leur sens. Et chez Deleuze et Guattari, ces agencements sont des mélanges ontologiques. L'agencement féodal, par exemple, qui est leur exemple canonique comprend des objets matériels (le château fort, le chevalier, son armure et ses armes, son cheval), des institutions et des discours (les lois et coutumes qui organisent l'ordre féodal, les cours de justice qui l'imposent, les rites qui le perpétuent, par exemple les joutes), mais aussi une répartition de l'espace, des habitus (gouvernant par exemple les rapports entre les sexes, ou entre seigneur et vilain) et enfin des textes (par exemple les poèmes d'amour courtois). Il me semble que l'avantage de l'étude des langues de spécialité est qu'elle nous contraint à pratiquer une linguistique externe au sens de Bourdieu, à ne jamais oublier que les textes que vous étudiez sont pris dans des agencements collectifs : les spécialistes décriront mieux que moi l'agencement collectif d'énonciation dont fait partie la langue des pilotes d'avion.

\section{Et où donc est la poétique de la langue de spécialité dans tout cela?}

Allons un peu plus loin. Il me semble que les langues de spécialité partagent avec la langue dite littéraire (je préfère dire: avec les jeux de langage qui constituent la littérature) une propriété importante, qui justifie qu'on leur applique le terme de " poétique ». Comme les langues littéraires, elles participent à la minoration de la langue standard. Ce concept appartient lui aussi à Deleuze et Guattari (il est exposé dans Mille Plateaux (1980) et dans Kafka (1975)): la majorité d'une langue standard n'est pas quantitative, mais résultat d'un rapport de forces. La langue majeure est ce qu'imposent certains appareils et institutions, même si bien peu de locuteurs la parlent réellement. Pour des raisons que l'on doit comprendre, mais que l'on doit aussi déplorer, nous enseignons l'« Anglais standard ", que nos étudiants minorisent allègrement. Car ce qui fait survivre une langue standard c'est qu'elle est sans cesse transposée sur le mode mineur par une multiplicité de dialectes, de styles, de registres et de jargons, qui la contestent et la font vivre. Ainsi l'anglais, langue de la globalisation, et de l'impérialisme, est minoré par la multiplication des New Englishes (au nombre desquels il faudra inévitablement un jour compter l'anglais de France), par ses propres dialectes régionaux (qui apparaissent maintenant jusqu'aux informations de la BBC, même si c'est aux marges, par exemple pour annoncer la météo), par les langues qu'elle a presque réussi à dévorer (par exemple le gallois - relisez la première page de Under Milk Wood pour voir ce qu'une gallisation poétique de l'anglais peut faire), mais aussi par les registres, par les styles littéraires (on parlera à juste titre de la « langue de Finnegan's Wake »). Parmi toutes 
ces minorités, qui produisent bien sûr la majorité des énoncés, il faut compter les langues de spécialité. C'est là qu'il y a une poétique de la langue de spécialité, comme chaque fois qu'il y a transposition en mineur de la langue majeure. Il y a donc un style des langues de spécialité, collectif comme tous les styles selon Deleuze, même s'ils sont pratiqués par ce qu'il appelle des « auteurs célibataires » comme Kafka. Certes, la définition qu'il donne du style du texte canoniquement littéraire ne conviendra pas ici : il est en effet caractérisé par une tension du langage vers ses limites, et vers le silence qui l'achève, au sens anglais comme au sens français du terme. Mais il y a certainement place pour une poétique de la langue de spécialité.

Comme vous êtes incrédules, comme Saint Thomas, vous attendez que je vous le prouve par un exemple. Pour être bref, je vais le faire indirectement, et terminer sur une pirouette : ce qui prouve qu'il y a une poétique de la langue de spécialité des scientifiques (souvenez-vous, c'est la seule que je connaisse un peu - Vidalenc me l'a apprise), c'est qu'elle est pastichable, et que Perec l'a pastichée dans Cantatrix Sopranica L. (1991). L'origine du texte est bien connue. Perec, de son métier bibliothécaire scientifique a monté ce canular - ce n'était pas le premier. Ses complices de l'Oulipo ont fini par le rendre public.

Regardons rapidement le début du texte (voir Annexe 2). Ce texte, naturellement possède toutes les caractéristiques que l'on peut attribuer, réellement ou fantasmatiquement, à un texte scientifique. Je dis "naturellement ", car c'est l'objet même du pastiche que de les relever. Et comme mon texte n'est pas tant un pastiche qu'un pastiche de pastiche, c'est-à-dire une parodie, son objet n'est pas seulement de les relever, mais de les marquer afin de les moquer. On fera donc la liste attendue des caractéristiques lexicales (le texte jargonne et polysyllabilise à l'envie), morpho-syntaxiques (pas d'embrayeurs, pas d'aspect en -ing, hormis quelques participes présents, pas de modaux, présence de formes passives: toutes les marques de l'impersonnel sont là), pragmatiques (abondance des références entre parenthèses, présence de guillemets - "yelling reaction», de sigles "YR » et d'italiques - «the more you throw tomatoes at Sopranoes, the more they yell ») et enfin stylistiques (des phrases d'une longueur digne de Proust, un goût immodéré pour l'hypotaxe et en même temps pour l'accumulation paratactique).

Moquées par la parodie, ces caractéristiques marquées finissent par pousser le jeu de langage de l'anglais scientifique jusqu'à ses limites (ce qu'il est, nous l'avons vu, une des définitions du style littéraire) : non seulement parce que le texte introduit un contenu loufoque dans un cadre formel sérieux, mais en ce que, utilisés par excès, et donc exploités, ces traits formels finissent par produire un effet de verve comique.

Ici, on peut dire que la parodie minore au carré, qu'elle minore une minorisation. Car la langue scientifique exploite les maximes de la langue standard, par exemple celles qui règlent la présence de l'énonciateur à son énoncé. Et la parodie minorise cette minorisation de multiples façons. J'en cite quelques-unes :

1. la parodie introduit, utilisant la présence obsédante des références entre parenthèses, un autre texte dans le texte: on se met en effet à lire les parenthèses à la suite, dans leur cohérence, de même que dans The Third Policeman, de Flann O'Brien (1967), on ne lit pas seulement l'histoire principale, on lit l'autre histoire, que racontent les notes en bas de page. Le rôle du second texte, qui est franchement comique, et bien plus inventif, et de tirer la barbichette du premier qui se donne les apparences du sérieux.

2. le second texte est fondé sur l'usage systématique du calembour et de tous les jeux que l'on peut pratiquer sur la forme des mots. Il n'introduit pas seulement des éléments 
d'encyclopédie qui font coq à l'âne, et rappellent le Je me souviens, autre texte célèbre de Perec (1978) (je me souviens de Roux et Combaluzier), il se livre à tous les louches trafics pour lesquels l'almanach Vermot fut jadis célèbre. Ce qui veut dire qu'il pratique systématiquement tous les jeux sur les mots que la langue scientifique s'interdit expressément, dont la langue littéraire raffole, et que les langues naturelles, en leur imperfection, trop souvent autorisent (cela s'appelle ambiguïté ou vague).

3. Cette rhétorisation par ce que j'ai ailleurs appelé le travail du reste (1990), du texte second, contamine le texte principal. Alors les listes se font litanies, et les mots se mettent à rimer («muscular, ventricular»). Alors s'introduisent les tropes les plus douteux, tel le chiasme (« puzzling tangle and tangling puzzle »). Si bien qu'un dialogue s'établit entre les deux textes, par lequel le premier finit par perdre son sérieux et à rire lui aussi ("digestive (Alka-Selzer)»).

41 Mais le texte scientifique parodié n'est minoré que parce qu'il est lui-même minorant, parce qu'il possède quelque chose qui relève du concept de style, et relève donc d'une forme de poétique, entendue non bien sûr comme description d'émotions esthétiques béates, mais comme analyse de phénomènes langagiers. Il y a bien une poétique des langues de spécialité.

\section{BIBLIOGRAPHIE}

Arasse, Daniel. 1999. L'Annonciation italienne, Paris : Hazan.

Deleuze, G. \& F.Guattari. 1975. Kafka, Paris : Minuit.

Deleuze, G. \& F. Guattari. 1980. Mille plateaux. Paris : Minuit.

Grice, H.Paul. 1989. Studies in the Way of Words. Cambridge, MA : Harvard University Press.

Lecercle, Jean-Jacques. 1990. The Violence of Language. Londres : Routledge.

Lecercle, Jean-Jacques. 1999. Interpretation as Pragmatics. Londres : Macmillan.

O’Brien, Flann. 1967. The Third policeman. Londres : Mc Gibbon \& Kee.

Perec, G. 1978. Je me souviens. Paris : Hachette.

Perec, G. 1991. Cantatrix Sopranica L. Paris : Seuil.

Sapphire. 1995. Push. Londres : Secker \& Warburg.

Vidalenc, Jean-Louis. 1995. Mémoire d'habilitation, Université de Paris X.

\section{ANNEXES}

Annexe 1 


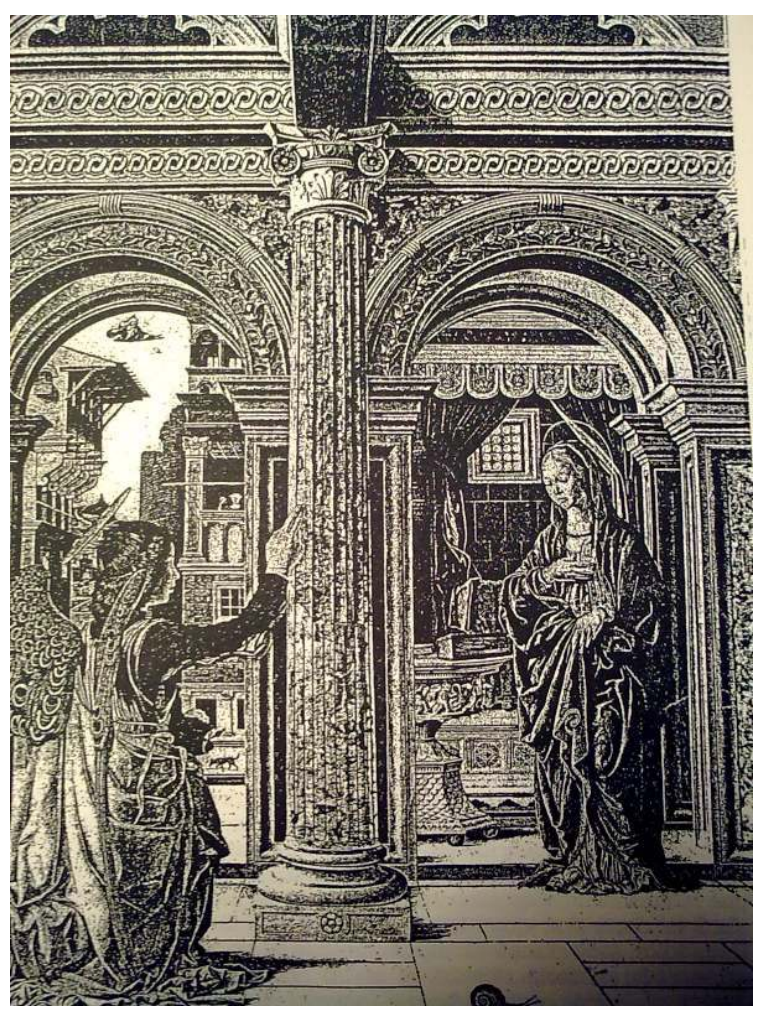

\section{Annexe 2}

G. Perec, Cantatrix sopranica L. (1991:13-15)

As observed at the turn of the century by Marks \& Spencer (1899), who first named the "yelling reaction" (YR), the striking effects of tomato throwing on Sopranoes have been extensively described. Although numerous behavioral (Zeeg \& Puss, 1931; Roux \& Combaluzier, 1932; Sinon et al., 1948), pathological (Hun \& Deu, 1960), comparative (Karybb \& Szyla, 1973) and follow-up (Else \& Vire, 1974) studies have permitted a valuable description of these typical responses, neuroanatomical, as well as neurophysiological data, are, in spite of their number, surprisingly confusing. In their henceforth latetwenties' classical demonstrations, Chuo \& Lai (1927a, b, c, 1928 a; b, 1929a, 1930) have ruled out the hypothesis of a pure facio-facial nociceptive reflex that has been advanced for many years by a number of authors (Mace \& Doyne, 1912; Paire \& Tairnelle, 1916; Sornette \& Billevayzé, 1925). Since that time, numerous observations have been made that have tried to decipher the tangling puzzle as well as the puzzling tangle of the afferent and/or efferent sides of the YR and led to the rather chaotic involvement of numberless structures and paths: trigeminal (Lowewenstein et al., 1930), bitrigeminal (von Aitick, 1940), quadritrigeminal (Van der Deder, 1950), supra-, infra-, and intertrigeminal (Mason \& Ragoun, 1960) afferents have been likely pointed out as well as macular (Zakouski, 1954), saccular (Bortsch, 1955), utricular (Malosol, 1956), ventricular (Tarama, 1957), monocular (Zubrowska, 1958), binocular (Chachlik, 1959-60), triocular (Strogonoff, 1960), auditive (Balalaiika, 1515) and digestive (Alka-Seltzer, 1815) inputs. Spinothalamic (Attou \& Ratathou, 1974), rubrospinal (Maotz \& Toung, 1973), nigrostriatal (Szentagothai, 1972), reticular (Pompeiano et al., 1971), hypothalamic (Hubel \& Wiesel, 1970), mesolimbic (Kuffler, 1969) and cerebellar (High \& Low, 1968) pathways have been vainly searched out for a tentative explanation of the YR organization and almost every part of the somesthetic (Pericoloso \&Sporgersi, 1973), motor (Ford, 1930), commissural (Gordon \& Bogen, 1974) and associative (Einstein et al., 1974) cortices have 
been found responsible for the progressive building up of the response although, up to now, no decisive demonstration of both the input and output of the YR programming has been convincely advanced.

\section{RÉSUMÉS}

La dialectique de la médiation et de l'immédiat dans les langues de spécialité est envisagée en commençant par un détour : l'analyse d'une Annonciation italienne de la Renaissance en termes d'effet de réel et de construction médiate de l'immédiateté. Cette illusion est ensuite décrite dans les deux domaines de la linguistique (à travers une critique de la philosophie du langage de Chomsky) et de la littérature (critique de l'illusion qui fait de l'auteur la source du sens du texte). La sortie de l'illusion passe par l'analyse d'un énoncé au centre d'un fait-divers, l'affaire Bentley, et une introduction au modèle ALTER de construction du sens. Elle passe aussi par le concept d'agencement collectif d'énonciation, emprunté à Gilles Deleuze. Ceci permet d'envisager une poétique de la langue de spécialité à partir de la parodie de Perec, « Cantatrix Sopranica L ».

The dialectics of mediation and immediacy in English for special purposes is approached via a detour: the analysis of an Italian Annunciation, in terms of reality effect and the mediated construction of an illusion of immediacy. That illusion is described in the two fields of linguistics (through a critique of Chomsky's philosophy of language) and literature (where the illusion that seeks the source of the text's meaning in the author is denounced). A way out of the illusion is found through the analysis of the utterance at the heart of a criminal case, the Bentley case, and the introduction of the ALTER model for the construction of meaning. It also involves the use of Deleuze's concept of collective assemblage of enunciation. Lastly, poetics in English for special purposes is evoked through an analysis of Perec's parody, "Cantatrix Sopranica L".

\section{INDEX}

Keywords : ambiguity, collective assemblage of enunciation, illusion of immediacy, interpellation, language game, linguistic conjuncture, mediation, minority, parody, reality effect, style

Mots-clés : agencement collectif d'énonciation, ambiguïé, conjoncture linguistique, effet de réel, illusion d'immédiateté, interpellation, jeu de langage, médiation, minorité, parodie, style

\section{AUTEUR}

\section{JEAN-JACQUES LECERCLE}

Jean-Jacques Lecercle est Professeur de littérature anglaise à l'université de Cardiff. Spécialiste de littérature victorienne et de philosophie du langage, il est entre autres l'auteur de La Violence du langage (PUF 1996), Interpretation as Pragmatics (Macmillan 1999), L'Emprise des signes (en collaboration avec R. Shusterman, Seuil 2002), Deleuze on Language (Palgrave 2002).

jlecercle@mageos.com 